\title{
Disease-Management-Programme im Risikostrukturausgleich: Überraschende Ergebnisse im Jahresausgleich 2004
}

Seit dem Jahr 2003 werden strukturierte Behandlungsprogramme (DMP) finanziell im Risikostrukturausgleich gefördert. Mit dem Jahresausgleich 2004 sind erstmals belastbare Aussagen über die finanziellen Auswirkungen möglich, da mit dem Programm für Diabetes mellitus Typ 2 ein flächendeckend laufendes Programm mit rund einer Million eingeschriebener Versicherter existiert. Es zeigt sich, dass es für Krankenkassen nicht nur auf die Zahl der Eingeschriebenen ankommt, sondern auch auf deren Altersstruktur.

\section{Frank Otto und Dirk Göpffarth}

\section{Zielsetzung der strukturierten Behandlungsprogramme}

Mit der Einbindung der strukturierten Behandlungsprogramme (DMP) in den Risikostrukturausgleich hat der Gesetzgeber eine besondere finanzielle Förderung dieser Versorgungsform angestrebt. Hiermit soll die Versorgung chronisch Kranker, die bislang von gleichzeitig bestehender Über-, Unter- und Fehlversorgung gekennzeichnet war (vgl. SVR 2001), verbessert werden. Seit dem Jahr 2003 laufen flächendeckend Programme für Diabetes mellitus Typ 2. Im Jahresausgleich 2004 wurden knapp 1 Million eingeschriebene Versicherte berücksichtigt, so dass zum ersten Mal genauere Aussagen über die finanziellen Folgen der Einbindung in den Risikostrukturausgleich gemacht werden können. Dabei besteht über die vielfältigen Wirkungs- und Kompensationsmechanismen des Risikostrukturausgleichs häufig Unklarheit. Im folgenden Beitrag sollen diese Mechanismen anhand der Rechnungsergebnisse des Jahresausgleichs 2004 für Diabetes mellitus Typ 2 auf Ebene der Kassenarten dargestellt werden.

\section{Ergebnisse des Jahresausgleichs 2004}

Durch die Einführung von eigenständigen Versichertengruppen für in DMPs eingeschriebene Versicherte werden Krankenkassen mit einem hohen Anteil chronisch Kranker über den Risikostrukturausgleich finanziell entlastet. Für einen eingeschriebenen Versicherten erhält die Krankenkasse einen höheren Beitragsbedarf als für einen nicht eingeschriebenen. Im Jahresausgleich 2004 betrug

Frank Otto und Dr. Dirk Göpffarth, Bundesversicherungsamt, Bonn der finanzielle Vorteil der Einschreibung durchschnittlich 1.288 Euro: Für einen eingeschriebenen Diabetiker erhielt eine Krankenkasse im Schnitt 4.390 Euro Beitragsbedarf pro Jahr, während für nicht eingeschriebene Versicherte mit derselben Alters- und Geschlechtsstruktur durchschnittlich 3.102 Euro zugewiesen wurden.

Diese Erhöhung des Beitragsbedarfs für DMP-Teilnehmer wird finanziert durch die Verminderung des Beitragsbedarfs, der den übrigen, nicht eingeschriebenen Versicherten zugewiesenen wird. Im Jahresausgleich 2004 betrug diese Absenkung im Durchschnitt 19 Euro. Eine Krankenkasse profitiert demnach von der Einschreibung eines eigenen Versicherten, die Einschreibung eines Versicherten einer anderen Krankenkasse bedeutet hingegen eine finanzielle Belastung. Zu diesen Änderungen des Beitragsbedarfes kommen die Auswirkungen der DMP-Programmkostenpauschalen in Höhe von 84,14 Euro je Versichertenjahr hinzu. Hierdurch sollen die Aufwendungen erstattet werden, die zusätzlich und unmittelbar im Zusammenhang mit der Entwicklung, Zulassung, Durchführung und Evaluation dieser Programme entstehen. Insgesamt werden so zusätzlich 82 Millionen Euro an die Krankenkassen ausgeschüttet, die über eine Anhebung des Ausgleichsbedarfssatzes von allen Krankenkassen finanziert werden.

Die finanziellen Auswirkungen der strukturierten Behandlungsprogramme auf die Transferzahlungen im Risikostrukturausgleich des Jahres 2004 sind der Tabelle 1 zu entnehmen. ${ }^{1}$ Die Tabelle zeigt zwar die Finanzströme aller DMPs, da aber im Jahr 2004 den 970.042 Versichertenjahren für Typ 2-Diabetiker nur 11.028 für Brustkrebs und 1.639 für Koronare Herzkrankheit gegenüber standen, sind diese Ergebnisse nahezu ausschließlich durch das DMP Diabetes geprägt. 
Tabelle 1: Finanzielle Auswirkungen der strukturierten Behandlungsprogramme im Risikostrukturausgleich 2004

\begin{tabular}{|l|c|c|c|c|}
\hline Kassenart & Beitragsbedarf $^{*}$ & VK-Pauschale* $^{*}$ & Gesamtwirkung $^{*}$ & BS-Effekt \\
\hline AOK & $39.964 .813,10$ & $13.807 .904,41$ & $53.772 .717,51$ & $-0,02$ \\
\hline BKK & $14.356 .310,20$ & $-7.523 .305,09$ & $6.833 .005,11$ & 0,00 \\
\hline BKN & $14.091 .524,37$ & $2.889 .824,74$ & $16.981 .349,10$ & $-0,09$ \\
\hline EAN & $-57.014 .228,01$ & $-6.961 .178,36$ & $-63.975 .406,38$ & 0,02 \\
\hline EAR & $-4.188 .400,25$ & $-934.956,83$ & $-5.123 .357,08$ & 0,02 \\
\hline IKK & $-5.268 .962,24$ & $-1.203 .355,08$ & $-6.472 .317,31$ & 0,01 \\
\hline SEE & $-1.941 .057,14$ & $-74.933,78$ & $-2.015 .990,92$ & 0,17 \\
\hline
\end{tabular}

* Angaben in Euro

Quelle: Eigene Berechnungen auf Grundlage von Bundesversicherungsamt (2005)

Ortskrankenkassen, Betriebskrankenkassen und die Bundesknappschaft profitieren per Saldo von den DMP, wohingegen Ersatzkassen, Innungskrankenkassen und die See-Krankenkasse finanzielle Einbußen hinnehmen müssen. Im folgenden soll aufgezeigt werden, welche Faktoren dieses Ergebnis bestimmen.

Tabelle 2: Anteil der in ein DMP eingeschriebenen Versicherten an allen Versicherten der jeweiligen Kassenart

\begin{tabular}{|l|c|}
\hline Kassenart & DMP-Anteil \\
\hline AOK & $1,81 \%$ \\
\hline BKK & $1,05 \%$ \\
\hline EAN & $3,82 \%$ \\
\hline EAR & $1,12 \%$ \\
\hline IKK & $0,76 \%$ \\
\hline SEE & $0,92 \%$ \\
\hline GKV & $0,42 \%$ \\
\hline
\end{tabular}

Quelle: Eigene Berechnungen auf Grundlage von Bundesversicherungsamt (2005)

\section{Erklärungsfaktoren der Transferströme}

\subsection{Einfluss der Einschreibequote}

Um zu verstehen, warum sich die Finanzwirkungen für die verschiedenen Kassenarten so stark unterscheiden, bietet es sich zunächst an, die Einschreibequote zu betrachten. Tabelle 2 gibt den Anteil der in ein DMP eingeschriebenen Versicherten an der Summe der Versichertentage aller Versicherten der jeweiligen Kassenart in Prozent an. ${ }^{2}$

Man sieht, dass die Knappschaft die höchste und die SeeKrankenkasse die niedrigste Einschreibequote aufweist. Auch die hohe Quote der Ortskrankenkassen ist ein Indiz für die Tatsache, dass die Finanzwirkungen der DMP von der Einschreibequote abhängen. Dieser Zusammenhang allein vermag jedoch nicht alle in Tabelle 1 angegebenen Effekte zu erklären: die Quote der Betriebskrankenkassen beispielsweise liegt noch unter der der Angestellten-Ersatzkassen. Dennoch führen die DMP für erstere zur Erhöhung und für letztere zur Absenkung des Beitragsbedarfs. Demnach spielt nicht nur der Anteil der Eingeschriebenen eine Rolle, sondern auch deren Struktur. Der noch von Häussler et al. (2005) unterstellte enge Zusammenhang zwischen Einschreibequote und Beitragsbedarfstransfer ist in Frage zu stellen. Deshalb soll nun der Einfluss der Altersstruktur auf die von den DMP verursachten Zahlungsströme analysiert werden.

\subsection{Einfluss der Altersstruktur}

Der finanzielle Vorteil der Einschreibung in Höhe von durchschnittlich 1.288 Euro hängt in seiner tatsächlichen Höhe von den übrigen Ausgleichsmerkmalen des Risikostrukturausgleichs ab, d. h. vom Alter, Geschlecht und 
dem Bezug einer Erwerbsminderungsrente. Abbildung 1 zeigt für Männer und Frauen ohne Erwerbsminderungsrente den Zusammenhang zwischen dem Alter und der Höhe des zusätzlichen Beitragsbedarfs für eingeschriebene Diabetiker. Da es nur sehr vereinzelt Typ 2-Diabetiker mit einem Alter von unter 30 Jahren gibt, wurden diese Werte nicht aufgezeigt. Aus dem Kurvenverlauf geht deutlich hervor, dass die Beitragsbedarfsvorteile mit zunehmendem Alter sinken. So bringt zum Beispiel ein 50-jähriger Diabetiker seiner Krankenkasse durch Einschreibung in ein strukturiertes Behandlungsprogramm einen mehr als doppelt so hohen Beitragsbedarfszuwachs wie ein 85-jähriger Diabetiker.

Die Gesamtwirkung der DMPs besteht aber nicht nur in der Erhöhung des eingeschriebenen Versicherten zugewiesenen Beitragsbedarfs, sondern wird ergänzt durch die gleichzeitig stattfindende Absenkung des Beitragsbedarfs für nicht eingeschriebene Versicherte; im Durchschnitt betrug diese 19 Euro. Abbildung 2 zeigt den Zusammenhang zwischen Alter und Absenkung des Beitragsbedarfes für nicht eingeschriebene Versicherte. Die maximale Ab- senkung erfolgt in der Altersklasse der 70-jährigen. Für jüngere und ältere Versicherte ist das Ausmaß der Absenkung geringer.

Damit wird deutlich, dass sowohl die Erhöhung des Beitragsbedarfs für DMP-Teilnehmer, als auch die Absenkung des Beitragsbedarfs für die übrigen Versicherten vom Alter abhängt. So erklärt sich auch, warum die Betriebskrankenkassen per Saldo von den DMP profitieren während die Ersatzkassen trotz höherer Einschreibequote schlechter gestellt sind. In den Alterskassen von 35 bis 55 Jahren beispielsweise kommt es zu einer relativ starken Erhöhung der Beitragsbedarfs (siehe Abbildung 1). Der Anteil der eingeschriebenen Diabetiker in diesen Altersklassen an allen eingeschriebenen Diabetikern liegt bei den Betriebskrankenkassen mit 16,8\% über dem der Versicherten der Angestellten-Ersatzkassen mit 14,1\%. Zudem wirkt sich die Absenkung bei letzteren stärker aus, da bei ihnen die von der Absenkung besonders betroffenen Altersgruppen stärker besetzt sind. Während bei den Angestellten-Ersatzkassen die nicht eingeschriebenen Versicherten zwischen 56 und 82 Jahren 27,1\% aller nicht eingeschriebenen Versicherten ausmachen, beträgt

\section{Abbildung 1: Beitragsbedarfsdifferenz zwischen eingeschriebenen Diabetikern und nicht eingeschriebenen Versicherten nach Alter}

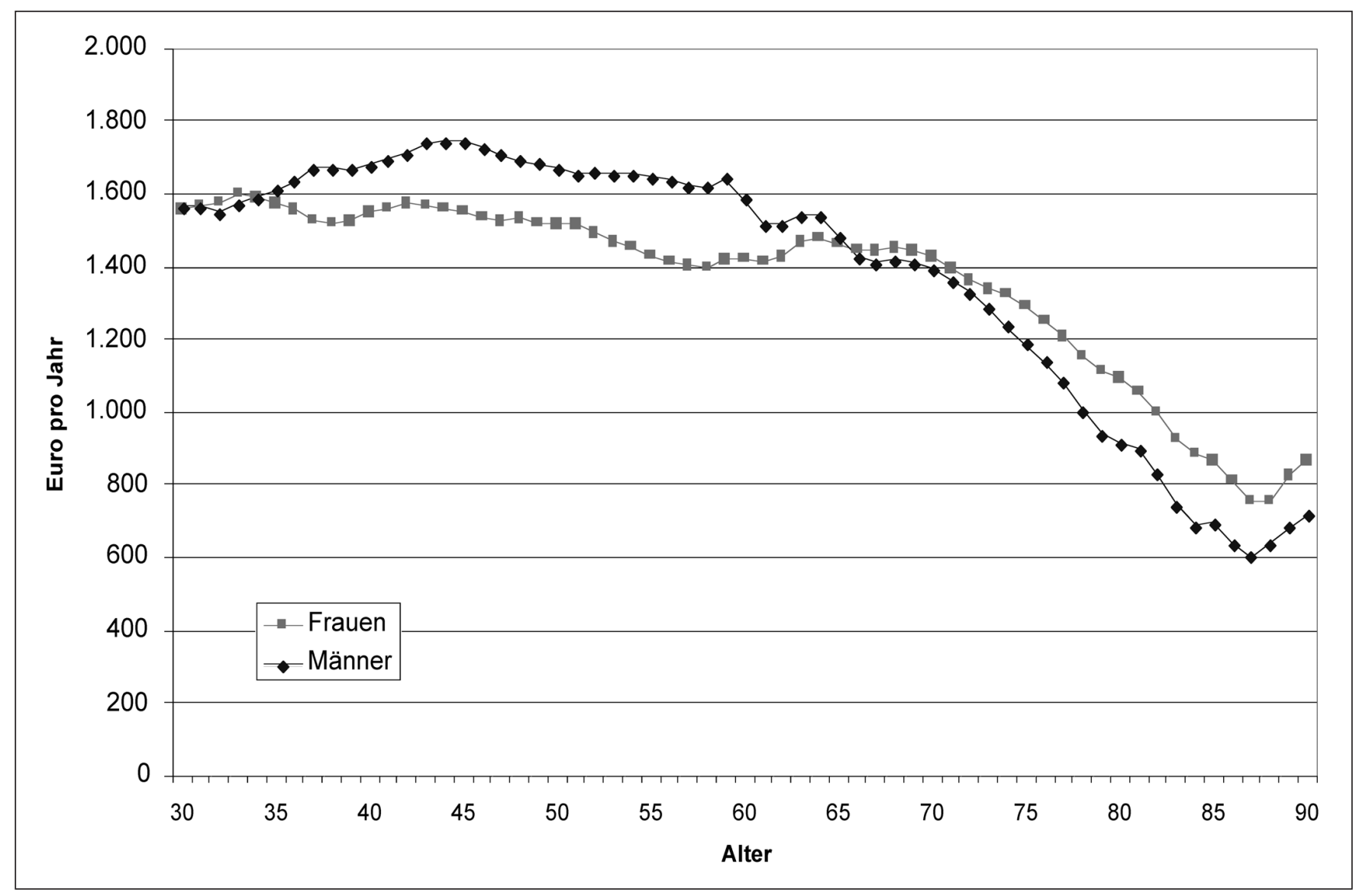

Quelle: Eigene Berechnungen auf Grundlage von Bundesversicherungsamt (2005) 
Abbildung 2: Beitragsbedarfsabsenkung für nicht eingeschriebene Versicherte nach Alter

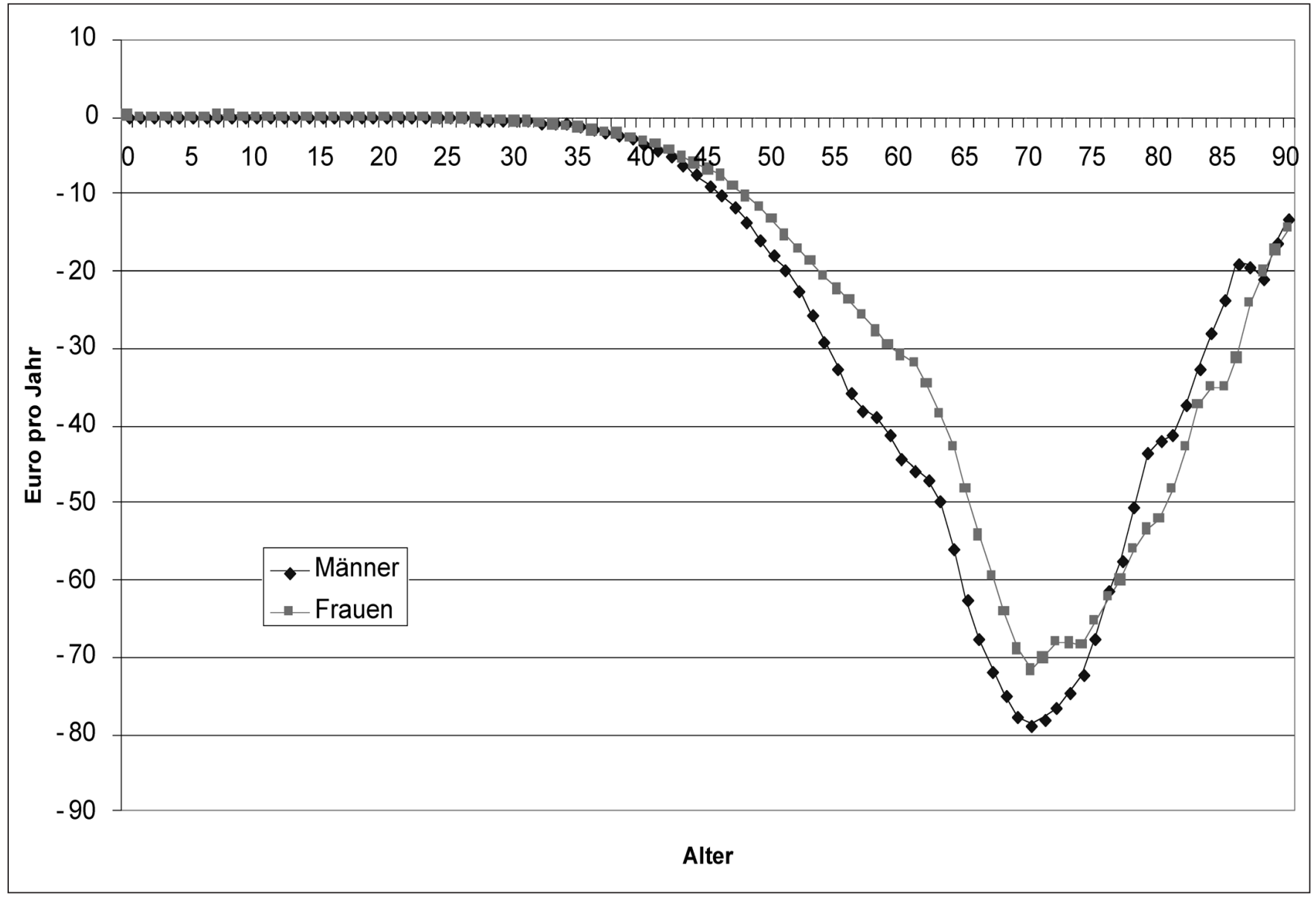

Quelle: Eigene Berechnungen auf Grundlage von Bundesversicherungsamt (2005)

die Quote bei den Betriebskrankenkassen nur 18,4\%. Der höhere Anteil der jüngeren Versicherten begünstigt die Betriebskrankenkassen.

Die Unterschiede in der Altersstruktur der eingeschriebenen Versicherten lassen sich auch in der folgenden Darstellung (Abbildung 3) erkennen. Für verschiedene Kassenarten ist der Anteil der in ein DMP eingeschriebenen Versicherten an allen Versicherten jeder Altersklasse aufgetragen, d.h. es handelt sich um die Einschreibequoten je Altersklasse. Das Rätsel der unterschiedlichen Wirkung der DMPs bei Betriebskrankenkassen und Ersatzkassen kann so gelöst werden: Obwohl die Angestellten-Ersatzkassen insgesamt eine geringfügig höhere Einschreibequote aufweisen (siehe Tabelle 2), liegt die Einschreibequote in jeder einzelnen Altersgruppe bei den Betriebskrankenkassen zum Teil deutlich über der der Ersatzkassen. Letztere erreichen die höhere durchschnittliche Einschreibequote nur dadurch, dass sie einen größeren Anteil Versicherter in den höheren Altersgruppen aufweisen, bei denen die Einschreibequote generell höher liegt. In weiten Teilen überschreitet die Einschreibequote je Altersgruppe bei den Betriebskrankenkassen sogar die der Ortskrankenkassen.

\section{Schlussfolgerungen}

Die Einschreibung in ein DMP lohnt sich finanziell immer für eine Krankenkasse, unabhängig vom Alter des Versicherten. Mit jüngeren Versicherten kann jedoch ein höherer Beitragsbedarfszuwachs realisiert werden. In wie weit damit auch eine kontraproduktive Selektion auf weniger schwer betroffene Fälle - wie von Häussler et al. (2005) anhand der Ergebnisse aus dem Jahresausgleich 2003 dargestellt - verbunden ist, kann mit den vorliegenden Zahlen nicht ermittelt werden. Gegenüber der zitierten Untersuchung ist kritisch anzumerken, dass die Daten des Jahres 2003 nicht nur, wie von den Autoren berücksichtigt, in Bezug auf das ambulante Leistungsgeschehen eine Fehlerfassung aufweisen, sondern auch eine Untererfassung der stationären Leistungsausgaben. Da die $\mathrm{Zu}-$ lassungen der DMPs in der Regel erst zum Jahresende 2003 erfolgten, gingen für die erfassten Versicherten nur kurze Versicherungsepisoden in die Berechnungen ein. Da eine Einschreibung zum Zeitpunkt eines stationären Aufenthaltes unwahrscheinlich ist und der kurze Zeitraum nach der Einschreibung nicht ausreichte, um die stationären Einweisungswahrscheinlichkeiten dieser Gruppe abzubil- 
den, waren auch die Ausgabenprofile im Krankenhaus im Jahresausgleich 2003 erheblich niedriger als im Jahresausgleich 2004.

Eine Krankenkasse mit einem hohen Anteil junger Versicherter ist darüber hinaus weniger stark von der durch die DMPs ausgelösten Profilabsenkung betroffen. Diese wirkt insbesondere bei Altersgruppen, die durch ein breites Morbiditätsspektrum gekennzeichnet sind. Es besteht die Gefahr, dass sich die Fehlanreize bei der Versorgung anderer (chronisch) Kranker, für die kein DMP vorgesehen ist, erhöhen (vgl. Wille und Resch 2005).

Nun ist das Alter ein Ausgleichsfaktor im Risikostrukturausgleich, der bewirken soll, dass Krankenkassen keine Vor- und Nachteile aufgrund der Altersstruktur ihrer Versicherten haben. Zwar vermindern die DMP die Anreize, eine gute Versorgung chronisch Kranker - bezogen auf das berücksichtigte Morbiditätsspektrum - zu unterlassen. Dennoch bleibt festzuhalten, dass die DMP auch neue Selektionsanreize schaffen, die die Wirkung anderer Ausgleichsfaktoren, wie hier am Beispiel Alter aufgezeigt, beeinträchtigen können. Als Form der stärkeren Morbiditätsorientierung des Risikostrukturausgleich bleiben die DMP damit den morbiditätsorientierten Klassifikationsmodellen (Reschke et al. 2004) unterlegen.

\section{Literatur}

Bundesversicherungsamt (2005): Daten zum Jahresausgleich 2004, www.bva.de, [Zugriff am 02.02.2006].

Häussler, Bertram; Eberhard Wille, Jürgen Wasem und Philipp Storz (2005): Diabetiker im Disease Management, Gesundheits- und Sozialpolitik, Heft 9-10/2005, S. 23-33.

Reschke, Peter; Stephanie Sehlen; Guido Schiffhorst; Wilhelm F. Schräder; Karl Lauterbach; Jürgen Wasem (2004): Klassifikationsmodelle für Versicherte im Risikostrukturausgleich, Bundesministerium für Gesundheit und Soziale Sicherung, Forschungsbericht 334, Bonn.

SVR (2001), Sachverständigenrat für die Konzertierte Aktion im Gesundheitswesen: Bedarfsgerechtigkeit und Wirtschaftlichkeit, Band III, Bonn.

Wille, Eberhard; Stefan Resch (2005): Risikoselektion trotz Risikostrukturausgleich?, in: Norbert Klusen, Christoph Straub und Andreas Meusch (Hg.): Steuerungswirkungen des Risikostrukturausgleichs, Baden-Baden, S. 13-36.

\section{Fußnoten}

1 Es werden folgende Abkürzungen verwendet: $\mathrm{AOK}=$ Allgemeine Ortskrankenkassen, BKK = Betriebskrankenkassen, BKN = Knappschaft, $\mathrm{EAN}=$ Angestellten-Ersatzkassen $(\mathrm{VdAK}), \mathrm{EAR}=$ Arbeiter-Ersatzkassen (AEV), IKK = Innungskrankenkassen, SEE = See-Krankenkasse.

2 Wie hoch die Einschreibequote im Sinne des Verhältnisses der eingeschriebenen Diabetikern zu allen Diabetikern liegt, kann anhand der Daten des Risikostrukturausgleichs nicht eingeschätzt werden. Für eine Abschätzung entsprechender Prävalenzraten siehe Häussler et al. (2005)

\section{Abbildung 3: DMP-Einschreibequote nach Alter}

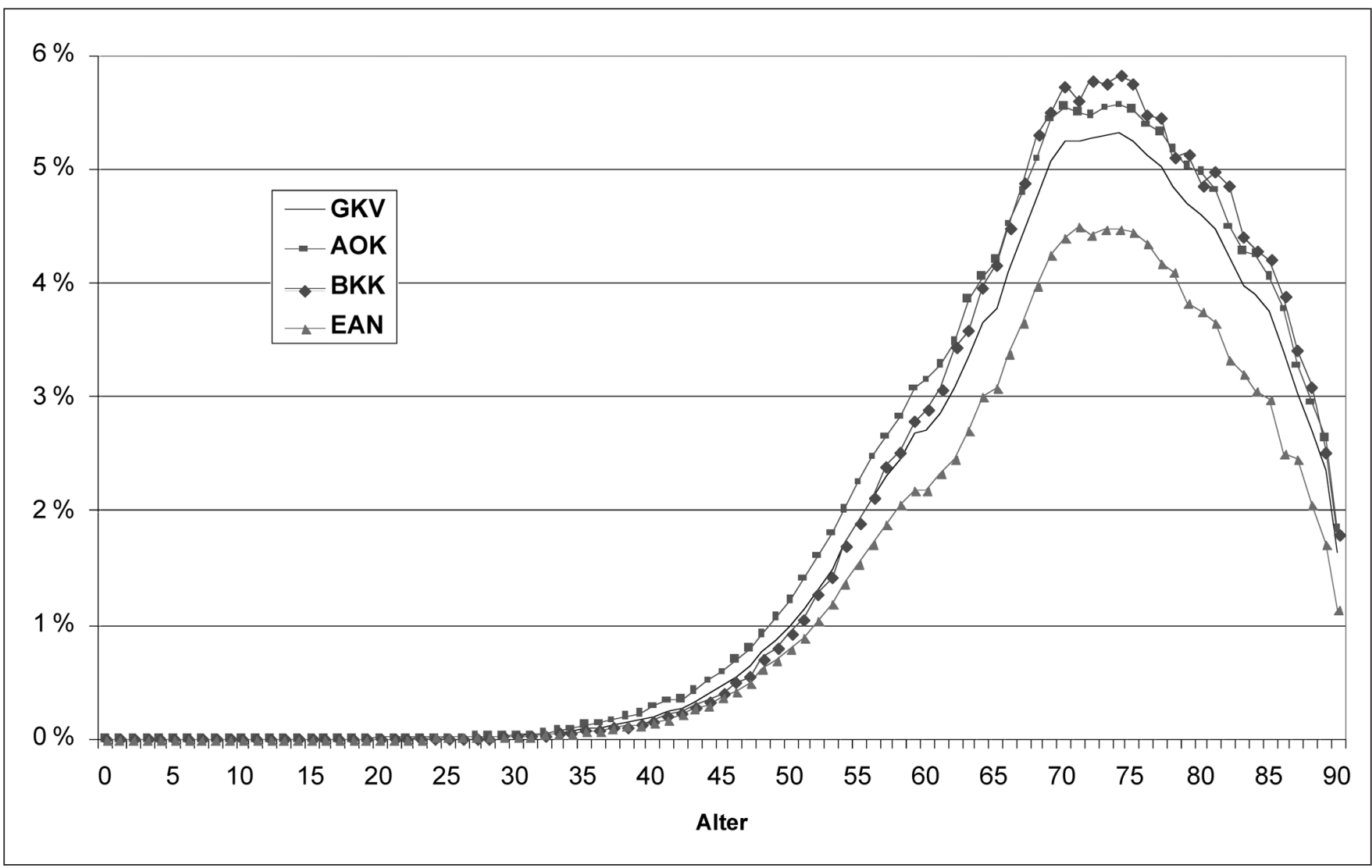

Quelle: Eigene Berechnungen auf Grundlage von Bundesversicherungsamt (2005) 